\title{
MicroscopyPioneers
}

\section{Pioneers in Optics: Albert A. Michelson}

\section{Eric Clark}

From the website Molecular Expressions created by the late Michael Davidson and now maintained by Eric Clark, National Magnetic Field Laboratory, Florida State University, Tallahassee, FL 32306

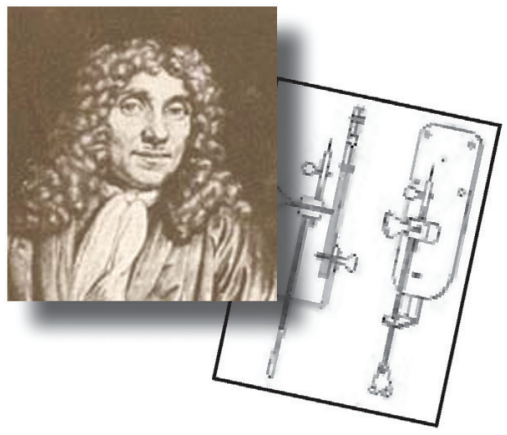

eclark@magnet.fsu.edu

\section{Albert A. Michelson \\ (1852-1931)}

The renowned physicist Albert Abraham Michelson was awarded the Nobel Prize in Physics in 1907. He spent a significant amount of his life trying to improve upon earlier scientific research and is often credited with the statement "The grand underlying principles have been firmly established ... further truths of physics are to be looked for in the sixth place of decimals." Michelson is most widely remembered for his experiments in which he proved that the hypothetical medium of light, known as "ether," did not exist, as well as his many attempts to accurately measure the speed of light.

Although Michelson was born in Strzelno, Prussia (now Poland), on December 19, 1852, his family moved to the United States when he was only 3 years old. Landing in New York, the Michelson clan soon traveled westward, eventually settling in San Francisco, California, where the elder Michelson became a successful merchant. At the age of 17, Albert Michelson entered the United States Naval Academy and excelled in his studies of the sciences, although he was a less than admirable sailor. Graduating in 1873, he began teaching science at the Naval Academy in 1875 and remained in the position for four years.

During the period of his military employment, Michelson started work on what would be a driving force in his life, the precise measurement of the speed of light. However, Michelson realized that he would need to know more about optics in order to be able to improve on the values previously obtained by others. He traveled to Europe with the intention to study the field and retired from the Navy in 1881. When he returned to North America, he established the most accurate value of the velocity of light available at that time, 299,853 kilometers per second. The measurement remained the best for almost an entire generation before he made it even more precise.

In 1883, Michelson became a physics professor at the Case School of Applied Science in Cleveland, Ohio, but continued in his penchant for research. In 1887, he conducted a series of experiments with Edward W. Morley to detect the existence of ether, a hypothetical all-pervasive medium that many scientists believed extended throughout the universe. Michelson developed an interferometer, a device that uses light wave interference to measure the differences in velocity of two light beams. The interferometer, using mirrors, splits a beam of light in two, sending them along perpendicular paths, and then brings them back together again. By analyzing any resulting interference fringes due to miniscule mismatches in the beams, very delicate measurements of differences in velocity of light can be made. Michelson intended to use the interferometer to measure the velocity of the Earth against the ether. If the Earth were traveling through a medium, then the speed of light traveling in the same direction as the Earth would be different than light traveling perpendicular to the direction of the Earth. In what may be the most famous null result ever obtained, Michelson and Morley found no interference fringes and, therefore, no evidence of ether.

Michelson accepted a professorship at Clark University in 1890 but left only a few years later to teach physics and become the first department head at the newly built University of Chicago. When World War I began, Michelson volunteered for military duty even though he was 65 years old and once again became a member of the United States Navy. Returning to Chicago in 1918, he redirected his studies toward the field of astronomy. Although Armand Fizeau was the first to suggest that interferometry could be used to measure the size of stars, Michelson was the first to actually use an interferometer to measure the size of a stellar body. In 1920, he used a six-meter interferometer attached to a 254-centimeter telescope to successfully measure the diameter of Betelgeuse (Alpha Orionis) at 386,160,000 kilometers.

Michelson died on May 9, 1931, but his research laid the groundwork for further discoveries in the twentieth century in the areas of the properties of light, space exploration, and atomic energy. In fact, in a tribute to Michelson published after his death, Albert Einstein praised him justly: "My honored Dr. Michelson, it was you who led the physicists into new paths, and through your marvelous experimental work paved the way for the development of the theory of relativity." 